OPEN ACCESS

Edited by: Shih-Min Wang National Cheng Kung University, Taiwan

Reviewed by:

Arturo Solis-Moya

Dr. Carlos Sáenz Herrera National Children's Hospital, Costa Rica

Raja Ahsan Aftab,

Taylor's University, Malaysia

${ }^{*}$ Correspondence:

Xiaoyan Dong

dongxy1515@126.com

Specialty section

This article was submitted to Pediatric Infectious Diseases,

a section of the journal

Frontiers in Pediatrics

Received: 28 May 2020 Accepted: 17 September 2021 Published: 12 October 2021

Citation:

Zhong H and Dong X (2021) Analysis of Clinical Characteristics and Risk Factors of Severe Adenovirus

Pneumonia in Children.

Front. Pediatr. 9:566797. doi: 10.3389/fped.2021.566797

\section{Analysis of Clinical Characteristics and Risk Factors of Severe Adenovirus Pneumonia in Children}

\author{
Haiqin Zhong and Xiaoyan Dong* \\ Department of Respiratory Medicine, Shanghai Children's Hospital, Shanghai Jiao Tong University, Shanghai, China
}

Objective: To analyze the clinical characteristics of adenovirus pneumonia (ADVP) in children and explore risk factors for severe ADVP.

Methods: Clinical data from 7,008 hospitalized children with community-acquired pneumonia and 211 with ADVP were retrospectively analyzed between July 2014 and June 2019. Eighty-six patients were diagnosed with severe pneumonia, and related risk factors were analyzed.

Results: ADVP accounts for 3.01\% (211/7008) of CAP in hospitalized children. Among 211 patients, 167 (64.9\%) children aged $1-5$ years old, and the onset was in winter and spring for 126 (59.7\%) children. All patients had cough, and 116 (92.8\%) patients with mild cases and 82 (95.4\%) patients with severe cases had varying degrees of fever. The duration of fever in the severe ADVP group and mild ADVP group was 7.3 and 5.4 days, respectively. The average hospital stays were 9.8 and 5.8 days, respectively. There was no significant difference in the levels of WBC and ESR between the two groups, but the levels of N\%, CRP, PCT and LDH in children with severe ADVP were significantly higher than those in the mild ADVP group. The univariate analysis showed that there were significant differences between the severe ADVP group and the mild ADVP group in $\geq 7$ days of fever and high $\lg E(P<0.05)$. There was no significant difference in sex, age, onset season, mycoplasma infection, bacterial infection between the two groups $(P>0.05)$. The multivariate logistic analysis showed that $\geq 7$ days of fever and high lgE were independent risk factors for severe ADVP $(P<0.05)$.

Conclusions: Children with severe ADVP have long fever duration, a strong inflammatory response and immune function disturbance. Fever duration ( $\geq 7$ days) and high IgE were independent risk factors for severe ADVP.

\section{Keywords: adenovirus, pneumonia, risk factors, children, clinical characteristics}

\section{INTRODUCTION}

Human adenovirus is well known pathogens that cause a variety of human illness, including upper respiratory tract illness, pneumonia, conjunctivitis, gastroenteritis and cystitis (1). Adenovirus $(\mathrm{ADV})$, a nonenveloped double-stranded DNA virus, is known to have more than 90 genotypes, with seven subgroups (A to G) (2). Adenovirus pneumonia (ADVP) is a common respiratory infectious disease in children, accounting for $4-10 \%$ of pneumonia cases in children (3). One-third 
of adenoviral pneumonia develops into severe pneumonia (4). Severe ADVP is characterized by respiratory system involvement, multiple system complications, high mortality, chronic airway and lung diseases, and severe mental and economic burden to family and society. In the present article, we report the characteristics of ADVP and provide an analysis of the clinical features and risk factors associated with severe ADVP cases in children.

\section{METHODS}

We conducted a retrospective case-control study combined with a descriptive analysis of clinical data and outcome among children admitted to department of respiratory medicine of Shanghai Children's Hospital over the period from July 2014 and June 2019. Previously healthy 7,008 male and female children between 1 month and 14 years who had been hospitalized for community-acquired pneumonia (CAP) were included in the study. ADVP was defined as clinician-diagnosed pneumonia and positive adenovirus antigen or IgM antibodies. The exclusion criteria of the study were asthma, endotracheal foreign body, known or suspected active tuberculosis, lung mycosis, pulmonary parasitoses, immunodeficiency, receiving immunosuppressive agents. Patient characteristics, including demographics, clinical presentation, laboratory examination and outcomes were extracted from electronic medical records.

Community-acquired pneumonia was defined as fever and cough with chest radiographic findings of lobar/bronchopneumonia or focal infiltrates. Fever was defined as a body temperature of $>37.5^{\circ} \mathrm{C}$. The duration of fever before hospitalization was provided by the caregivers, whereas the duration of fever after hospitalization was collected from medical records. The diagnosis of severe pneumonia was any one of the following: sustained high fever $\left(>39^{\circ} \mathrm{C}\right)$ for more than 5 days, accompanied by a frequent and severe irritating cough; Respiratory rate $\geq 70$ breaths/min (infant), Respiratory rate $\geq 50$ breaths $/ \mathrm{min}$ ( $\geq 1$ year old); Severe respiratory failure $\left(\mathrm{PaO}_{2} / \mathrm{FiO}_{2}<250\right)$; Rapidly progressing lung shadow with multiple- or single-lobar/segment consolidation; Requirement for mechanical ventilation; Requiring intensive care. Admission to ICU was also based on clinical assessment by pediatric senior medical staff and was indicated if there was clinically significant respiratory distress, dehydration, hypotension and/or suspicion of encephalopathy.

Adenovirus detection was done via (1) Adenovirus antigen testing: respiratory tract samples, including tracheal aspirates, bronchoalveolar lavage, induced sputum and nose swabs or nasopharyngeal aspirates, were collected and adenovirus antigen was detected by immunofluorescence test (D3 Ultra DFA Respiratory Virus Screening \& ID Kit, Diagnostic Hybrids, Inc.). (2) Specific IgM antibody detection: Two $\mathrm{ml}$ of venous blood were taken, and serum specific IgM antibodies of common respiratory pathogens were detected by indirect immunofluorescence assay (Respiratory Tract Profile (IgM), EUROIMMUN Medical Diagnostics (China) Co., Ltd.). In addition, other microbiologic tests for bacteria,
TABLE 1 | Comparison of inflammatory indicators between the mild ADVP group and the severe ADVP group.

\begin{tabular}{lcccc}
\hline Items & Mild ADVP & Severe ADVP & $\boldsymbol{t} / \boldsymbol{Z}$ value & $\boldsymbol{P}$ \\
\hline WBC $\left(\times 10^{9} / \mathrm{L}\right)^{\mathrm{a}}$ & $9.15 \pm 5.10$ & $9.68 \pm 5.13$ & 0.734 & 0.464 \\
$\mathrm{~N}^{\mathrm{a}}$ & $48.79 \pm 19.10$ & $55.74 \pm 20.33$ & 2.529 & $0.012^{*}$ \\
$\mathrm{CRP}(\mathrm{mg} / \mathrm{L})^{\mathrm{a}}$ & $19.39 \pm 19.32$ & $28.49 \pm 38.05$ & 2.283 & $0.023^{*}$ \\
$\mathrm{ESR}(\mathrm{mm} / \mathrm{h})^{\mathrm{a}}$ & $44.91 \pm 26.83$ & $47.72 \pm 28.28$ & 0.731 & 0.466 \\
$\mathrm{PCT}(\mathrm{ng} / \mathrm{mL})^{\mathrm{b}}$ & $0.52 \pm 1.08$ & $1.69 \pm 4.88$ & -2.484 & $0.013^{*}$ \\
$\mathrm{LDH}(\mu \mathrm{mol} / \mathrm{L})^{\mathrm{b}}$ & $333.12 \pm 94.09$ & $563.11 \pm 424.29$ & -6.005 & $0.000^{*}$
\end{tabular}

at-text; ${ }^{b}$ Mann-Whitney $U$ test; ${ }^{*} P<0.05$.

viruses and mycoplasma pneumoniae were requested when clinically indicated.

Statistical analysis was performed using the SPSS statistical software program, version 20 (IBM Corporation, Armonk, NY, USA). The measurement data are expressed as the mean \pm standard deviation $(\bar{x} \pm s)$. The count data were expressed as a percentage or rate. The chi-square test was used for categorical data, and the $t$-test and Mann-Whitney $U$ test were used for continuous data. Binary logistic regression analysis was used to examine risk factors that were significant in the univariate analysis. $P<0.05$ was considered statistically significant.

\section{RESULTS}

\section{General Information}

A total of 7,008 hospitalized children with CAP were investigated and 211 children with ADVP were included in the retrospective study according to the inclusion criteria. ADVP accounts for $3.01 \%(211 / 7,008)$ of CAP in hospitalized children. Among 211 children with ADVP, the ratio was of boys and girls 1.25:1(117:94). The age of onset was 3 months to 8 years. Thirty patients (14.2\%) were under 1 year of age, 76 patients $(36.0 \%)$ were 1-3 years old, 61 patients $(26.2 \%)$ were 3-5 years old, and 44 patients (20.9\%) were older than 5 . The cases occurred throughout the year, including 57 cases $(27.0 \%)$ in spring (March to May), 53 (25.1\%) in summer (June to August), 33 (15.7\%) in autumn (September to November), and 68 (32.2\%) in winter (December to January). Winter and spring were the peak seasons ( $n=125,59.2 \%)$. A total of 86 patients $(37.7 \%)$ were diagnosed with severe pneumonia, and 125 (62.3\%) were diagnosed with mild pneumonia. There was no significant difference in sex, age or onset season between the two groups.

\section{Clinical Features}

Varying degrees of fever were found for 116 (92.8\%) patients with mild ADVP and 82 (95.4\%) patients with severe ADVP. The duration of fever in the mild groups lasted $<10$ days for 95 patients $(76.0 \%)$ and longer than 10 days for 21 patients (16.8\%). The duration of fever in the severe groups lasted $<10$ days for 58 patients $(67.4 \%)$ and longer than 10 days for 24 patients (27.9\%). The average durations of fever for the severe ADVP group and mild ADVP group were 7.3 and 5.4 days, respectively. The average hospital stays were 9.8 and 5.8 days, respectively. The 
duration of fever and hospital stays of the mild ADVP group were less than those of the severe group.

All patients had cough, and 45 patients $(21.3 \%)$ also had wheezing. Moreover, 56 patients (65.1\%) had wet rales, and 21 patients with severe ADVP (24.4\%) had a wheezing sound in their lungs. 75 patients $(60.0 \%)$ had wet rales, and 24 patients with mild ADVP (19.2\%) had a wheezing sound.

\section{Laboratory Tests}

There was no significant difference in the white blood cell count (WBC) and erythrocyte sedimentation rate (ESR) between the two groups, but the neutrophil (N\%), C-reactive protein (CRP), procalcitonin (PCT) and lactate dehydrogenase (LDH) levels in children with severe ADVP were significantly higher than those in the mild ADVP group, as shown in Table $\mathbf{1}$.

\section{Radiographic Examination}

Chest X-ray examination was performed in all 211 children, and patchy opacity (177 cases, $83.9 \%$ ), patchy consolidation (118 cases, $55.9 \%$ ) and pleural effusion (70 cases, $33.2 \%)$ were most common.

Chest CT examination was performed in 86 severe ADVP cases, and patchy opacity of bilateral lungs (80 cases, 93.0\%), lung consolidation and/or atelectasis (67 cases, $77.9 \%$ ) were most common. The range of pulmonary lesions involved one lobe in eight cases $(9.3 \%)$, six lobes in two cases $(7.0 \%)$, more than or equal to three lobes in 72 cases $(83.7 \%)$.

\section{Risk Factors for Severe ADVP \\ The Univariate Analysis}

There were significant differences between the severe ADVP group and the mild ADVP group in $\geq 7$ days of fever and high IgE $(P<0.05)$. There was no significant difference in sex, age, onset season, mycoplasma infection, bacterial infection, between the two groups $(P>0.05)$, as shown in Table 2 .

\section{Multivariate Logistic Analysis}

Binary logistic regression analysis was used to examine risk factors that were significant in the univariate analysis. The independent variables were assigned values: $\geq 7$ days of fever: No $=0$, Yes $=1$; high IgE: No $=0$, Yes $=1$. Whether severe ADVP occurred or not was taken as the response variable. The results showed that $\geq 7$ days of fever and high IgE were independent risk factors for severe ADVP $(P<0.05)$, as shown in Table 3.

\section{DISCUSSION}

$\mathrm{ADV}$ is one of the severe types of community-acquired pneumonia in children. ADVP accounts for $3.01 \%$ of CAP in hospitalized children in our study. The early clinical manifestations of ADVP lack specificity, and most of them begin with fever and cough. It is difficult to distinguish from other respiratory tract infectious diseases, and relevant pathogenic detection methods are needed. A total of $92.8 \%$ of mild and 95.4\% of severe cases of ADVP were associated with varying degrees of fever. Patients with severe cases had a longer duration of fever. The disease progresses rapidly in a short period of
TABLE 2 | Univariate analysis between the mild ADVP group and the severe ADVP group.

\begin{tabular}{|c|c|c|c|c|c|}
\hline Characteristics & & $\begin{array}{c}\text { Mild } \\
\text { ADVP } \\
(n= \\
125)\end{array}$ & $\begin{array}{c}\text { Severe } \\
\text { ADVP } \\
(n=86)\end{array}$ & $\chi^{2}$ value & $P^{a}$ \\
\hline \multirow[t]{2}{*}{ Sex } & Male & 67 & 50 & 0.425 & 0.514 \\
\hline & Female & 58 & 36 & & \\
\hline \multirow[t]{4}{*}{ Age } & $\leq 1$ years & 16 & 14 & 0.808 & 0.847 \\
\hline & $\begin{array}{l}1 \sim 3 \\
\text { years }\end{array}$ & 44 & 32 & & \\
\hline & $\begin{array}{l}3 \sim 5 \\
\text { years }\end{array}$ & 38 & 23 & & \\
\hline & $>5$ years & 27 & 17 & & \\
\hline \multirow[t]{4}{*}{ Onset season } & Spring & 33 & 25 & 2.397 & 0.494 \\
\hline & Summer & 32 & 20 & & \\
\hline & Autumn & 23 & 10 & & \\
\hline & Winter & 37 & 31 & & \\
\hline \multirow[t]{2}{*}{ Days of fever } & $\geq 7$ & 39 & 43 & 7.579 & $0.006^{*}$ \\
\hline & $<7$ & 86 & 43 & & \\
\hline \multirow{2}{*}{$\begin{array}{l}\text { Mycoplasma } \\
\text { infection }\end{array}$} & Yes & 57 & 40 & 0.017 & 0.896 \\
\hline & No & 68 & 46 & & \\
\hline \multirow[t]{2}{*}{ Bacterial infection } & Yes & 23 & 19 & 0.436 & 0.509 \\
\hline & No & 102 & 67 & & \\
\hline \multirow[t]{2}{*}{ High IgE } & Yes & 63 & 58 & 6.049 & $0.014^{\star}$ \\
\hline & No & 62 & 28 & & \\
\hline
\end{tabular}

${ }^{a}$ chi-square tests; ${ }^{*} P<0.05$.

TABLE 3 | Multivariate logistic analysis of severe ADVP.

\begin{tabular}{|c|c|c|c|c|c|c|c|}
\hline $\begin{array}{l}\text { Independent } \\
\text { variables }\end{array}$ & nt $B$ & SE & Wald $\chi^{2}$ & $d f$ & $P$ & OR & $95 \% \mathrm{Cl}$ \\
\hline $\begin{array}{l}\geq 7 \text { days } \\
\text { of fever }\end{array}$ & 0.801 & 0.294 & 7.412 & 1 & $0.006^{\star}$ & 2.227 & $1.251 \sim 3.964$ \\
\hline High IgE & 0.723 & 0.297 & 5.927 & 1 & $0.015^{\star}$ & 2.061 & $1.151 \sim 3.689$ \\
\hline
\end{tabular}

${ }^{\star} P<0.05$.

time. Symptoms and signs of the respiratory system, including cough, wheezing, and rales, appeared obviously. Early etiological detection is the main method for the diagnosis of ADVP.

ADVP is more likely to occur in children aged 6 months to 5 years. As infants and young children grow, the number of maternal antibodies gradually decreases in the body, but their respiratory and immune systems are still not fully developed. Moreover, low local mucosal immunity contributes to a high incidence of adenoviral pneumonia in this age group (5). In this study, $79.1 \%$ of the children were under 5 years of age, which was consistent with domestic and foreign reports $(6,7)$. However, there was no significant difference in age between the two groups. Moreover, the two groups showed no significant difference in the onset season of ADVP, which was relatively high in winter and spring. Duan et al. (8) investigated the prevalence of ADV infection in children with community-acquired pneumonia 
(CAP) in 12 hospitals in nine provinces (municipalities directly under the central government) in China, and the incidence of ADV infection peaked in children aged $1 \sim 3$ years in Northern China, while there was no significant difference in the positive detection rate among age groups in Southern China. The season with the highest ADV detection rate in northern China was winter, and in southern China, it was spring and summer, suggesting that the onset ages and peak seasons may vary from region to region.

The levels of N\%, CRP, PCT and LDH in children with severe ADVP were significantly higher than those in the mild ADVP group. The relationship between N\%, CRP, PCT and the severity of ADVP has been confirmed by many studies (9-12). LDH is found in all animal tissues, and the highest activity is found in the liver, followed by the heart, skeletal muscle, kidney, lung and tumor. When the lung tissue is obviously damaged, the serum LDH level could be increased. In many diseases, it is a reliable indicator of severity and prognosis. Wen et al. (13) showed that serum LDH was higher in children with respiratory infection than in the healthy control group and was also higher in the pneumonia group than in the upper respiratory infection group. This study suggested that LDH elevation may be useful for the early identification of severe ADVP. In addition, Patel et al. (14) observed that adenovirus causes a greater increase in TNF- $\alpha$ than other viruses. Sun et al. (15) found a significant increase in IL6 levels in children with severe adenovirus infection, which was positively correlated with the severity of infection. Zheng et al. (16) found that the higher the level of IL-8 in children with ADVP, the worse the prognosis was. Therefore, there is a strong inflammatory process in severe ADVP.

Mixed mycoplasma infection in patients with ADVP was more common than mixed bacterial infection. But there was no significant difference in mycoplasma infection and bacterial infection between the two groups. The results indicated that for children with ADVP in this area, the occurrence of severe pneumonia is related to their physical fitness and immune function. Considering that children with poor physical fitness and low immune function are more likely to have mixed infection, the mixed infection was balanced in the two groups.

Literature has reported that the imaging manifestations of adenovirus pneumonia in children are mainly multiple segmental consolidation, atelectasis and patchy opacity in both lungs (17). More than three lobes of pulmonary lesions are involved in 72 cases $(83.7 \%)$ of severe ADVP. The main clinical features of cluster consolidation are centripetal distribution and high density. Some clinical studies have shown that the main reason for this clinical feature is related to the basic pathology of ADVP. When a patient is infected with ADV, the ADV will spread to the lung parenchyma along the airway and through the bronchus. When the degree of the lesion involves the bronchioles, it can affect the alveoli of the patient and further develop into lung consolidation.

Both of the univariate and multivariate logistic analysis suggested that there was significant difference in $\geq 7$ days of fever and high IgE between the two groups. Different studies show different risk factors for severe ADVP. It may be related to the different people, regions, environment, climate, and contents of comparisons. Jin et al. (18) found that age from 6 months to 2 years, nutritional anemia and immunodeficiency were independent risk factors for severe ADVP. Huang et al. (19) found that congenital airway dysplasia, history of previous surgery, and hemoglobin $<90 \mathrm{~g} / \mathrm{L}$ in children with ADVP had a greater association with severe disease. Xie et al. (20) showed that HADV load, age, and fever duration were risk factors for ADVP severity. Cheng et al. (21) demonstrated that patients who present with underlying neurologic diseases, chronic lung diseases, and malnutrition have higher incidences of ADVP and that ADV-7 infection more frequently develops into severe pneumonia. Overall, children with young age, long fever duration, underlying diseases and complications are more likely to develop severe ADVP.

At this point, the mechanism of severe adenovirus infection is unknown, although it is believed to be related to ADV itself and the ADV-induced inflammatory response and immune dysfunction. In this study, the IgE levels of ADVP were different in the mild and severe ADVP groups, and the severity of pneumonia was positively correlated with the IgE levels of ADVP. Abnormally high IgE levels are thought to be a marker of immune disorders $(22,23)$. At present, there are few studies on the correlation between ADV infection and $\operatorname{IgE}$ levels. Adenovirus, Mycoplasma pneumoniae, influenza virus and other infections could increase serum total IgE levels in children with respiratory tract infection (24). IL-4 promotes the production of IgG and $\operatorname{IgE}$ by B cells, so an abnormally high level of IL-4 may increase the IgE level in children with adenovirus infection (25), which may lead to inflammatory changes in the respiratory system. Yang et al. (26) showed that $\operatorname{IgE}$ and dsDNA-IgE in BALF may contribute to lung injury caused by HADVs, especially in severe cases and elevated dsDNAIgE may serve as an indicator of severity in children with HADVs pneumonia. Ye et al. (27) demonstrated that the P1 protein of Mycoplasma pneumoniae can induce the production of P1-specific IgE. Zhang et al. (28) found that the severity of respiratory syncytial virus (RSV) bronchitis in children was positively correlated with serum IgE level. The complexity of immunopathology and individual differences mean that ADV infection in children present a variety of disease processes, and immune dysfunction is an important pathogenesis that affects prognosis. We should pay more attention to the disease condition of ADVP patients with high IgE and select glucocorticoid or gamma globulin for immunoregulatory intervention therapy while controlling infection.

Our study also had limitations. First, the type of adenovirus is related to the severity of the disease and it was not detected in this retrospective study. Early identification is helpful for clinicians to identify severe cases and avoid delayed treatment. Second, this study discussed the correlation with IgE in acute infections and imply IgE was elevated in ADV infection. The results needed to be validated in more clinical cases and animal experiments. Besides, IL-4 may increase the IgE level. Data on IL-4 in patients were lacking in this study. Therefore, in the subsequent study, we could collect more data and analyze the relationship between the IgE level and IL-4, and it is best to study the mechanism involved on molecular biology level. 


\section{CONCLUSIONS}

Severe ADVP has a strong inflammatory reaction process, and immune dysfunction. Fever duration ( $\geq 7$ days) and high IgE are risk factors for severe ADVP. The detection of serum total IgE in children with ADVP not only provides a basis for the diagnosis and treatment of secondary allergic disease in children but also helps to judge the severity of the disease in order to provide early immune intervention and reduce inflammatory reactions. More attention should thus be paid to such cases.

\section{DATA AVAILABILITY STATEMENT}

The original contributions presented in the study are included in the article/supplementary material, further inquiries can be directed to the corresponding author.

\section{REFERENCES}

1. Radke JR, Cook JL. Human adenovirus infections: update and consideration of mechanisms of viral persistence. Curr Opin Infect Dis. (2018) 31:2516. doi: 10.1097/QCO.0000000000000451

2. Jobran S, Kattan R, Shamaa J, Marzouqa H, Hindiyeh M. Adenovirus respiratory tract infections in infants: a retrospective chart-review study. Lancet. (2018) 391:S43. doi: 10.1016/S0140-6736(18)30409-4

3. Fu FX, Tang Z, Ye Z, Mo S, Tian X, Ni K, et al. Human adenovirus type 7 infection causes a more severe disease than type 3. BMC Infect Dis. (2019) 19:36. doi: 10.1186/s12879-018-3651-2

4. Lu MP, Ma LY, Zheng Q, Dong LL, Chen ZM. Clinical characteristics of adenovirus associated lower respiratory tract infection in children. World $J$ Pediatr. (2013) 9:346-9. doi: 10.1007/s12519-013-0431-3

5. Berciaud S, Rayne F, Kassab S, Jubert C, Corte MF, Salin F, et al. Adenovirus infections in Bordeaus University Hospital 2008-2010: clinical and virological features. J Clin Virol. (2012) 54:302-7. doi: 10.1016/j.jcv.2012.04.009

6. Liu Q, Xie LY, Zhang B, Zhong LL, Yu T, Zeng SZ, et al. Epidemiological investigation of adenovirus pneumonia in children in Hunan province. Chin Pediatr Emerg Med. (2019) 26:752-7. doi: 10.3760/cma.j.issn.1673

7. Li L, Woo YY, de Bruyne JA, Nathan AM, Kee SY, Chan YF, et al. Epidemiology, clinical presentation and respiratory sequelae of adenovirus pneumonia in children in Kuala Lumpur, Malaysia. PLoS ONE. (2018) 13:e0205795. doi: 10.1371/journal.pone.0205795

8. Duan YL, Zhu Y, Xu BP Li CC, Chen AH, Deng L, et al. Multicenter study of human adenovirus infection in pediatric community-acquired pneumonia in China. Chin J Pediatr. (2019) 57:27-32. doi: 10.3760/cma.j.issn.0578-1310.2019.01.008

9. Rajkumar V, Chiang CSM, Low JM, Cui L, Lin RTP, Tee NWS, et al. Risk factors for severe adenovirus infection in children during an outbreak in Singapore. Ann Acad Med Singap. (2015) 44:50-9.

10. Chen J, Chen X, Tan XR, Liu DH Li XF, Zhang XX. Adenovirus pneumonia with hemophagocytic lymphohistiocytosis in children: a clinical analysis of 7 children. Zhongguo Dang Dai Er Ke Za Zhi. (2020) 22:749-54. doi: 10.7499/j.issn.1008-8830.19 12148

11. Liu JL, Xu F, Zhou H, Wu XJ, Shi LX, Lu RQ, et al. Expanded CURB65: a new score system predicts severity of community-acquired pneumonia with superior efficiency. Sci Rep. (2016) 6:22911. doi: 10.1038/srepr 22911

12. Zhydkov A, Christ-Crain M, Thomann R, Hoess C, Henzen C, Werner $Z$, et al. Utility of procalcitonin, C-reactive protein and white blood cells alone and in combination for the prediction of clinical outcomes in community-acquired pneumonia. Clin Chem Lab Med. (2015) 53:55966. doi: 10.1515/cclm-2014-0456

\section{ETHICS STATEMENT}

The studies involving human participants were reviewed and approved by the Ethics Committee of Shanghai Children's Hospital. Written informed consent from the participants' legal guardian/next of kin was not required to participate in this study in accordance with the national legislation and the institutional requirements.

\section{AUTHOR CONTRIBUTIONS}

HZ collected the patient's information, summarized the data, and wrote the paper. XD was a major contributor in revising the manuscript. Both authors read and approved the final manuscript.

13. Wen CB, Luo B, Liu QZ, Liang JL. Changes of serum uric acid, lactate dehydrogenase and alpha-hydroxybutyrate dehydrogenase in children with respiratory tract infection. China Medicine and Pharmacy. (2019) 9:657. doi: 10.3969/j.issn.2095-0616.2019.04.022

14. Patel JA, Nair S, Ochoa EE, Huda R, Roberts NJ, Chonmaitree T.Interleukin$6^{-174}$ and tumor necrosis factor $\alpha^{-308}$ polymorphisms enhancecytokine production by human macrophages exposed to respiratory viruses. J Interferon Cytokine Res. (2010) 30:917-21. doi: 10.1089/jir.2010. 0033

15. Sun J, Xiao Y, Zhang M, Ao T, Lang S, Wang J. Serum inflammatory markers in patients with adenovirus respiratory infection. Med Sci Monit. (2018) 24:3848-55. doi: 10.12659/MSM.910692

16. Zheng LL, Xu HY, Ying DH, T. lymphocyte subset level in children with severe adenoviral pneumonia and its clinical significance. Zhejiang Medical Journal. (2018) 40:1702-4. doi: 10.12056/j.issn.1006-2785.2018.40.15.2018-591

17. Han BK, Son JA, Yoon HK, Lee SL. Epidemic adenoviral lower respiratory tract infection in pediatric patients: radiographic and clinical characteristics. AJR Am J Roentgenol. (1998) 170:1077-80. doi: 10.2214/ajr.170.4.9530062

18. Jin DD, Zhou WF, Li Y, Kong XX, Tian JM. Characteristics of mixed infection of adenovirus pneumonia in children and risk factors of severe cases. J Clini Pulmon Med. (2019) 24:1747-50. doi: 10.3969/j.issn.1009-6663.2019.10.001

19. Huang CG, Chen L, Song YD. Characteristics and risk factors of infection with other pathogens of severe adenovirus pneumonia in children. J Hebei Medical University. (2019) 40:1221-5. doi: 10.3969/j.issn.1007-3205.2019.10.025

20. Xie L, Zhang B, Zhou J, Huang H, Zeng S, Liu Q, et al. Human adenovirus load in respiratory tract secretions are predictors for disease severity in children with human adenovirus pneumonia. Virol J. (2018) 15:123. doi: 10.1186/s12985-018-1037-0

21. Cheng JL, Peng CC, Chiu NC, Weng LC, Chiu YY, Chang L, et al. Risk Factor Analysis and molecular epidemiology of respiratory adenovirus infections among children in Northern Taiwan, 2009-2013. J Microbiol Immunol Infect. (2017) 50:418-26. doi: 10.1016/j.jmii.2015.08.006

22. Williams KW, Milner JD, Freeman AF. Eosinophilia associated with disorders of immune deficiency or immune dysregulation. Immunol Allergy Clin North Am. (2015) 35:523-44. doi: 10.1016/j.iac.2015. 05.004

23. Magen E, Schlesinger M, David M, Ben-Zion I, Vardy D. Selective IgE deficiency, immune dysregulation, and autoimmunity. Allergy Asthma Proc. (2014) 35:e27-33. doi: 10.2500/aap.2014.35.3734

24. Liu HJ, Dou M, Chen H, Chen J, Pan XJ, Shen LS. The correlation between atypical respiratory pathogens infection and serum total IgE levels. Int J Lab Med. (2015) 36:2480-4. doi: 10.3969/j.issn.1673-4130.2015. 17.011

25. Li Y, Gao Q, Yuan X, Zhou M, Peng $\mathrm{X}$, Liu $\mathrm{X}$, et al. Adenovirus expressing IFN- $\lambda 1$ (IL-29) attenuates Allergic airway 
inflammation and airway hyperreactivity in experimental asthma. Int Immunopharmacol. (2014) 21:156-62. doi: 10.1016/j.intimp.2014. 04.022

26. Yang DY, Lu BT, Shi TT, Fan HF, Zhang DW, Huang L, et al. Total and double-stranded DNA-specific immunoglobulin $\mathrm{E}$ in bronchoalveolar lavage fluid of children with human adenovirus pneumonia. J Infect Chemother. (2020) 26:986-91. doi: 10.1016/j.jiac.2020. 04.024

27. Ye Q, Mao JH, Shu Q, Shang SQ. Mycoplasma pneumoniae induces allergy by producing P1-specific immunoglobulin E. Ann Allergy Asthma Immunol. (2018) 121:90-9. doi: 10.1016/j.anai.2018. 03.014

28. Zhang RF, Liang X, Chen D, Guo L, Yang H, Xu Y, et al. Expression levels and clinical significance of serum $25(\mathrm{OH})$ D3 and immunoglobulin $\mathrm{E}$ in children with respiratory syncytial virus bronchiolitis. International Journal of Laboratory Medicine. (2019) 40:162-5. doi: 10.3969/j.issn.1673-4130.2019. 02.009
Conflict of Interest: The authors declare that the research was conducted in the absence of any commercial or financial relationships that could be construed as a potential conflict of interest.

Publisher's Note: All claims expressed in this article are solely those of the authors and do not necessarily represent those of their affiliated organizations, or those of the publisher, the editors and the reviewers. Any product that may be evaluated in this article, or claim that may be made by its manufacturer, is not guaranteed or endorsed by the publisher.

Copyright (c) 2021 Zhong and Dong. This is an open-access article distributed under the terms of the Creative Commons Attribution License (CC BY). The use, distribution or reproduction in other forums is permitted, provided the original author(s) and the copyright owner(s) are credited and that the original publication in this journal is cited, in accordance with accepted academic practice. No use, distribution or reproduction is permitted which does not comply with these terms. 